\title{
Research and Exploration on the Application of Large data in Modern Swine Industry
}

\author{
Yongmou Hua and Shuli Zhao \\ Beijing Agricultural Vocational College 102442
}

Keywords: Scale; Pigs; Big data; Dynamic management

\begin{abstract}
China is the world's largest pig country, the number of livestock and meat production are ranked first in all countries, but China is far from a big pig. The main reason is the lack of sound technical support system and imperfect market information system. This paper introduces large data into the dynamic management of swine industry, this paper analyzes the purpose of introducing large data management, puts forward the main construction content, expounds the problems needing to be solved, expounds the application of large data dynamic management technology of information, collects the large amount of data in the chain of prenatal, production and postpartum, predicts the market price, reduces the risk of breeding, and ensures the safety Improve production efficiency, so that the pig industry in China from the big pig into a powerful pig.

Along with our country in intensive livestock production, scale and specialized level unceasing enhancement, the livestock not only promote the rapid development of our country agriculture and rural economy, also provides consumers with meat, eggs, milk and so on security, plenty of agricultural and sideline products, greatly enriched the people table and basket. In particular, the pig industry has become the world's largest pig country, with the largest amount of stocks and meat. However, our country is far from being a pig country, because of the lack of perfect technical support system. Second, the market information system is not sound. This often causes the market volatility, the pig industry is difficult to develop smoothly. In particular, there are no pollution-free pigs. The state has standards, certification system and management system, but there is no supervision system, no policy support, no price advantage. Therefore, farmers are not motivated, consumers are not trustworthy, management has no tracking mechanism, and the country has no accurate data statistics. Therefore, big dynamic data management technology, using information collection before, during or after the chain of large amounts of data, analysis, mining, development and utilization, not only can the pollution-free pig, all-round, full coverage in the whole process of management, and to make an accurate prediction of production of live pigs breeding stock for master aquaculture, prediction markets, reduce the risk of breeding, to ensure the safety of products, improve production efficiency.
\end{abstract}

\section{The Significance of Applying large Data Dynamic Management}

\section{Application of large Data is the Inevitable Requirement of Industrial Development}

With the deepening of informatization, "Internet of things" and "Internet + " have become "important productive forces" to promote the rapid development of the world economy. It is said that "lack of data resources to talk about industry; Lack of data thinking, no future!" It can be said that future enterprises must be "data-driven enterprises". "data" will be an increasingly important asset for enterprises, which will completely change the concept of enterprise assets and the process of capital accumulation.

The pig industry is concerned with the lives of millions of people, which is related to the national CPI. And there are millions of farmers raising pigs, how to realize the accurate master data information, to grasp the industry at home and abroad market, reduce the risk of breeding and monitor the production process, to ensure product quality, these are not human factors can achieve, must use informatization means to achieve timely, comprehensive and accurate understanding and mastering the pig industry development basic situation, make clear, data base and the situation. Therefore, the application of big data to realize dynamic management is an inevitable trend of industrial development. 


\section{Subvert the Thinking Mode of Traditional Aquaculture Industry.}

Big data is applied to pig breeding, fully grasp the basic information of the industry, including the number of breeding and production of basic information, market information, the purpose is to focus on data, using the data, through the cloud of huge data statistics, analysis and research, and make the industry to achieve dynamic, informatization and scientific management. Therefore, whether it is concerned with the market price data, or the production data of pig farms. Or according to the development of the whole pig breeding data regulation, and according to the production data to trace the source of the pork to safeguard the interests of consumers, etc., are all depend on data to produce a new model of decision-making, to change a data-driven intelligent manufacturing, this is the traditional subversion of thinking mode of the animal husbandry, fundamentally solve the blindness and randomness existed in traditional pig breeding and empirical, continuously improve the scientification and informatization level of pig industry.

\section{Raise the level of Aquaculture Production.}

With the big data management platform, the daily management of pig farms can be monitored through the establishment of electronic files, and the production environment of pigs, such as feed, water, lighting, ventilation and health. At the same time, the physiological index, production index, epidemic prevention and feeding situation of pigs were recorded in detail. By using clear data for research, analysis, judgment, since as the basis to guide the production, including nutrition, epidemic prevention disinfection, disease diagnosis and treatment, etc., using the software system makes management more delicate and efficient. For example, the application of feed automatic feeding system can reduce a lot of waste. In the process of feeding, the pig can be automatically identified by the ear object, and the production performance of the pig can be inquired into by the computer, and different feeding amount is provided for individual differences. At the same time, we can judge the condition of pigs according to their intake. In addition, through dynamic management, accurate records of different Numbers of sows in different physiological condition, age structure, and the production performance, etc., to sow in a just, pregnancy, childbirth, and to match, and so on and so forth also record clear, sows are always in a state of optimization and efficient production status. Therefore, in the future, the core competitiveness of pig farms is not only good varieties, but accurate data statistics and analysis.

\section{The Production Information of Traceability Products.}

Use big data management, can fully monitor the process of production of live pigs, each pig production information, as well as individual pig production process are all files, in the process of production input material, immunization, disease diagnosis and treatment of such information will be clearly documented, from birth, slaughter quarantine, packaging sales have electronic file tracking process, such as the sale of meat product with bar code number on traceability identification, traceability identification number, the corresponding relationship between pig between and can confirm the purchased through database website pork products are properly qualified inspection and quarantine treatment. It can also be traced back to pig production enterprises, suppliers of inputs and slaughter and quarantine, etc., so that the whole production process can be monitored to ensure the safety of the production process. At the same time, consumers can understand the production process and ensure that the people can eat pork.

\section{The Main Content of Dynamic Management of Big Data.}

To establish a central database of pig health breeding information, and to conduct unified collection and centralized management of data on feeding, immunization, epidemic prevention and drug use of each pig in each farm. At the same time, a complete chain system of pork safety traceability is established, so that the problem of pork can be retroactively tracked from the market. The establishment of a true, complete and unified standard database should include the following three aspects: first, the development and establishment of a unified data management platform; Secondly, the management mechanism should be established step by step, including hardware input, personnel training and system establishment. Thirdly, data collection and analysis; Finally, the use of data is used to guide production, trace product information and ensure product quality. 


\section{Establish a Digital Management Platform.}

The safety of pork mainly includes feeding, slaughtering and processing and sales. If you are required to establish a unified central database covering three links. Once found disease pork, toxic pork, etc., which endanger the safety of the food events, can trace specific farms through the central database system, as well as the epidemic prevention and quarantine personnel find out specific reason and responsibility to personnel, the timely and effective corrective and processing. Moreover, consumers can understand the production, slaughtering and quarantine information of the products by tracing code. Development, therefore, to establish a big data management platform of live pigs, pig production, slaughter quarantine, packaging, storage and transportation, segmentation marketing, hazard analysis and critical control points of big data management, to ensure that the breeding, slaughtering, transportation and sales thoroughly safety; Using the digital identification technology, the purpose is to live pigs and pork products are identified to establish a quality traceability system of live pigs, such as breeding, slaughtering, processing and sales identity transformation and in the process of information transmission. It mainly includes production information, slaughtering and quarantine information, sales information and insurance information in the production process.

Farm Information System. The large-scale pig farms should establish electronic archives, which requires the unified collection and centralized management of the feeding, immunization, epidemic prevention and drug use of each pig in each farm. A record production basic information: including immune, quarantine, disease and down-stream recording system, reflect the immune, quarantine, major disease occurrence, feed, pharmaceutical and other inputs into source, name, quantity, investment of time and so on, as the pork product quality tracing the source of information. Second is piggery environmental parameter acquisition system through sensors to temperature, humidity, illumination, wind speed and ammonia concentration, automatic temperature control system, automatic water supply system, automatic ventilation system, automatic control system of harmful gases, and other, realize scientific, automation, informatiization.

Slaughter and Quarantine Information System. China is a populous country and a major producer of meat and food. People's consumption of meat is not only "appetizing", but also "taste" is getting higher and higher. Therefore, the establishment of the slaughter information system is the key to ensure the people's "tongue safety". It is also an important means to avoid slau ghtering and slaughtering and slaughtering dead animals, injecting water or injecting other substances and feeding "lean meat" into the market. It is also necessary to improve the management level and production efficiency of slaughtering enterprises. The information system shall include information such as the purchase and admission of pig pigs, static monitoring, pre-slaughter quarantine, slaughtering and processing, simultaneous quarantine, sales and settlement, logistics distribution and so on.

Traceability Information System. Traceability of sales information is primarily "the ability to track the history and use or location of a commodity or behavior through a registered identification code". Traceability information is the unique identification of each batch of products that have been recorded, and the history of the product is traced through its one-to-one correspondence with the tracked objects. On this basis, the establishment of the pig product traceability system, the data collection should include the whole process of food production chain, from origin and production information, to slaughter and processing process, until the storage and transportation sales each link, to provide consumers with accurate and detailed information about products. It can also help enterprises determine the flow of products and facilitate the tracking and management of products. To this end, the traceability system of animal products was first established within the eu. Experts predicted that in the industrial chain related to animal products, animal products "traceability" of mandatory management is inevitable, for the future development of the "safe meat" development plan of the ministry of commerce of the state council, has made a traceability system is an important issue.

Insurance and Harmless Treatment System. Pig insurance has a "high risk, high cost and high pay", the characteristics of insurance because pigs point more noodles wide, insurance companies need to invest a lot of manpower material resources, most parts of pig insurance covers all difficult 
to achieve. In addition, the insurance company is not an anti-epidemic law enforcement agent. In practice, it is often difficult to verify the amount of insurance coverage, difficult judgment of the target information, and the difficulty in the execution of harmless treatment. Therefore, the use of big data for dynamic management, through effective monitoring and tracking harmless treatment of dead livestock, greatly improved the work efficiency. At the same time, we can avoid the fraud protection, the number of dead pigs, the indiscriminate throwing of dead pigs and the cheap purchase of dead pigs, so as to eliminate the flow of dead animals from the source to the market and the people's table. Recuperated 2015 Beijing fangshan district and anwar insurance company have common development and in the region to promote the use of information technology means to survey the aquaculture insurance claims and risk control, has obtained the remarkable effect, become the whole city and even the "three rural" service new luminescent spot in the country.

\section{Data Collection and Analysis.}

Data Collection. Data collection mainly two parts, one is the basic conditions of farms and production, including the address, the surrounding environment, facilities and harmless treatment, the amount of data, output data, inputs data, data, and epidemic prevention data, clinical data, insurance, etc.; The second part is the slaughterhouse slaughter quarantine, due to the large scale pig farms are basically adopt all in all out batch management, even the farmers or free-range households small-scale farming in sent to a slaughterhouse for fixed-point slaughtering and processing. In slaughterhouses acquisition pig-breeding information, therefore, can be collected according to the production batch, but slaughter and quarantine process involves many links, each link should establish a information of data to collect, configure corresponding collection equipment, information collection of standards. For the detection of harmful bad information to the central slaughtering information database for traceability processing; The unqualified pork products are eliminated in a timely manner and processed safely; The qualified pork products can be traced back to the information mark and entered the sales link.

Data Analysis. The application of big data is not only the volume of mass data, but also the result of obtaining correct data and scientific data analysis. At present, the management software of pig farm is more the record and summary of pig farm data than the analysis and decision of pig farm data. Dynamic management of big data, collecting mass data, the purpose is through data analysis and processing, building a network of production and marketing price linkage to guide farmers science and arrange production, evading market risk, traces the product information. For example, the periodic cycle of pig breeding is the "pig cycle". The increase in pork prices leads to the increase in supply, causing the price of meat to fall. A fall in the price of meat has created a supply shortage, pushing up the price of meat. Must rely on science to solve "pig cycle", relying on big data research and analysis on the relationship between the market demand and production of live pigs, and to provide the necessary decision-making information for the breeding, reduce due to the breeding of the impact of changes in the economic cycle.

\section{Use Big Data Dynamic Management to Solve the Problem.}

Using big data dynamic management has been made possible and necessary, it can realize breeding way from the original extensive to standard fine breeding, which changes the farm management from experience management to information management. However, it is urgent to solve several problems in the real implementation of big data and cloud computing.

\section{Integrate Integrated Software Development Resources.}

At present, the big data has already begun to explore use in aquaculture, the current data have developed the use of tools everywhere, such as pigs, together with Beijing fangshan district agriculture bureau and anwar insurance insurance system, which can realize rapid and accurate pig insurance claims and harmless treatment; And god bless the cloud pig via "pig" management software, can be in pig breeding, nutrition, environment and biological safety generate huge amounts of data for intelligent processing, quickly find out the related data and profit, generate intelligent pig business each link of the decision support module, reduce pig production management blind dependence on experience. But these are only operators for development and 
design, the production requirements of a link to that fails to cover the breeding process, also can integrate sharing data resources, may even cause unnecessary waste of resources and data of the disorder. Therefore, we must start from the competent administrative department for agriculture, accomplish the top-level design, comprehensive statistics, whole course monitoring, to fully monitor every pig's life, of the pig all can accurately grasp the situation.

\section{Increase Financial Support.}

Driving big data dynamic management requires a lot of hardware and software conditions as well as operational management costs, which is the cost that farmers and farms are unwilling to spend and can not bear. Therefore, it must be coordinated and led by the government and supported and invested by the government. Hardware facilities such as computer terminal, scanner, ear javelin, ear tag, etc. And the development of software management system also requires a lot of money, need a lot of management personnel to implement management at the same time, need to advance technical training, also needs the support of relevant expenses, during the operation of this is to use the necessary premise and guarantee for big data.

\section{Establish a Government-led Management Mechanism.}

China's pig breeding is complicated, although intensive farming develops rapidly, but it is still the main body from rural areas. So to make a big promotion data management, the first to start from the large-scale farms, pollution-free farming enterprises, in particular, they must also have the qualification to apply for and certification, can be big data management as apply for certification is one of the conditions or requirements, equipped with hardware and software conditions, a week to farmers or aquaculture enterprises to carry out the relevant data collection. Secondly, the promotion and coverage of farmers are gradually established. But no matter which way, must be managed by the competent department, establish national, provincial, township, village level management network, each district and even administrative village must have full-time management personnel. At the same time, establish relevant systems and mechanisms to ensure the accurate collection of information and ensure the use of data to guide the production and traceability of product quality.

\section{References}

[1] zheng jiukun. Application of big data in animal husbandry technology promotion service [J]. China animal husbandry, 2015, (01)

[2] chang li, zhang zhijin. Discussion on big data and industry development of animal husbandry [J]. China animal husbandry and veterinary digest, 2015, (11).

[3] wen xiaojiang. Strategic significance and cooperative mechanism of agricultural big data research [J]. Higher agriculture education, 2013, (11)

[4] Peng L, Xiaoping Z. Social Stratification and Cooperative Behavior in Spatial Prisoners' Dilemma Games [J]. PLOS ONE, 2015, 10(7): e0131005.

[5] Information on http://hn.ifeng.com/a/20171218/6236493_0.shtml

[6] D. Wang: Study on the Development of Tourism Educaiton in Jilin Province (MS., Northeast Normal University, China 2011), p.2. 